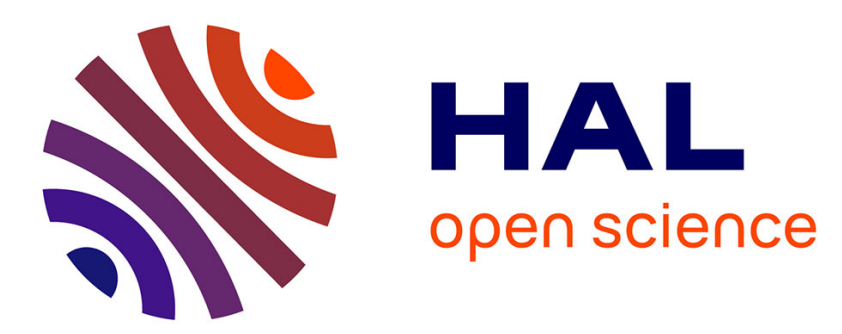

\title{
Genetic algorithms-based inversion of multimode guided waves for cortical bone characterization
}

\author{
N Bochud, Q Vallet, Y Bala, Hélène Follet, J-G Minonzio, P Laugier
}

\section{To cite this version:}

N Bochud, Q Vallet, Y Bala, Hélène Follet, J-G Minonzio, et al.. Genetic algorithms-based inversion of multimode guided waves for cortical bone characterization. Physics in Medicine and Biology, 2016, 61 (19), pp.6953. 10.1088/0031-9155/61/19/6953 . hal-01371942

\section{HAL Id: hal-01371942 \\ https://hal.sorbonne-universite.fr/hal-01371942}

Submitted on 29 Sep 2016

HAL is a multi-disciplinary open access archive for the deposit and dissemination of scientific research documents, whether they are published or not. The documents may come from teaching and research institutions in France or abroad, or from public or private research centers.
L'archive ouverte pluridisciplinaire HAL, est destinée au dépôt et à la diffusion de documents scientifiques de niveau recherche, publiés ou non, émanant des établissements d'enseignement et de recherche français ou étrangers, des laboratoires publics ou privés. 


\title{
Genetic algorithms-based inversion of multimode guided waves for cortical bone characterization
}

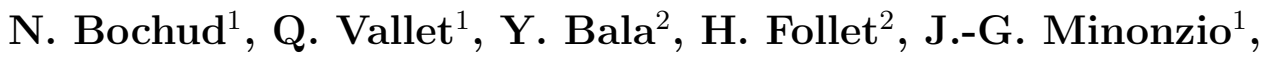 \\ and P. Laugier ${ }^{1}$ \\ ${ }^{1}$ Sorbonne Universités, UPMC Univ Paris 06, CNRS UMR 7371, INSERM UMR \\ S1146, Laboratoire d'imagerie biomédicale, 15 rue de l'école de médecine, F-75006, \\ Paris, France \\ ${ }^{2}$ Université de Lyon, Université Claude Bernard Lyon 1, INSERM UMR1033, Lyon, \\ France \\ E-mail: nicolas.bochud@upmc.fr \\ February 2016
}

\begin{abstract}
Recent progress in quantitative ultrasound exploit the multimode waveguide response of long bones. Measurements of the guided modes, along with suitable waveguide modeling, have the potential for inferring strength-related factors such as stiffness (mainly determined by cortical porosity) and cortical thickness. However, the development of such model-based approaches is challenging, particularly due to the multiparametric nature of the inverse problem. Current estimation methods in the bone field rely on a number of assumptions for pairing the incomplete experimental data with the theoretical guided modes (e.g., semi-automatic selection and classification of the data). The availability of an alternative inversion scheme that is user-independent is highly desirable. Thus, this paper introduces an efficient inversion method based on genetic algorithms using multimode guided waves, in which the mode-order is kept blind. Prior to the evaluation on bone, our proposal is validated on laboratory-controlled measurements on isotropic plates and bonemimicking phantoms. Results show that the model parameters (i.e., cortical thickness and porosity) estimated from the measurements on a few ex vivo and in vivo human radii are in good agreement with the reference values derived from X-ray-based techniques.
\end{abstract}

\section{Introduction}

Elastic guided waves (GWs) is one of the most promising quantitative ultrasound (QUS) technique under development for characterizing cortical bone. Several experimental studies have evidenced that long cortical bones as radius and tibia could act as natural waveguide for ultrasound despite attenuation, irregular geometry and heterogeneous material properties $[1,2,3]$. Measurement of GWs is particularly attractive because they propagate throughout the bone thickness and may thus answer to the attempt of determining multiple bone properties, such as cortical thickness and material properties, 
which are in turn relevant indicators of the bone biomechanical competence [4]. Cortical loss observed with aging, which results in cortical thinning, porosity increase, impaired mechanical properties and increased fracture risk [5], is expected to change the propagation characteristics of the guided modes. Consequently, GWs-based approaches have a strong potential for yielding a more complete characterization of cortical bone than does conventional X-ray bone densitometry [6].

A GWs-based approach is typically associated with the measurement of long bones in the so-called axial transmission (AT) configuration, which exploits the propagation of GWs in the cortical shell along the main bone axis. AT techniques have been extensively used for measuring the velocity of the first arriving signal (FAS) in bone. Investigations based on FAS have been both conducted in laboratory conditions on phantoms or ex vivo [7, 8], and in clinical studies [9, 10, 11, 12, 13, 14, 15]. Additional slower guided modes have been investigated over the past decade, and it has been shown that a fundamental flexural guided mode (equivalent to the Lamb $A_{0}$-mode for a plate) could also be identified with this technique [16]. A multimode approach combining measurement of two modes at specific frequencies has also been shown to provide a possibility for discerning contributions of the material properties and the cortical thickness [17]. Despite these encouraging results, little attention has yet been paid to cortical bone characterization using multimode GWs. In particular, measurements of higher-order guided modes could increase our confidence in the inferred ultrasound bone biomarkers but yield a challenge of solving multiparametric inverse problems [18].

In that vein, three Lamb modes (identified as $A_{0}, S_{0}$ and $A_{1}$ ) were experimentally observed in an ex vivo study [3]. Estimates of the Young's moduli of three ox bone samples were then obtained by fitting an isotropic plate model (with given thickness and density) to measured phase velocities using a least-mean-square algorithm. In another related study, Ta et al. [19] identified the thickness of a bovine tibia by manually fitting measured phase velocities of the three first cylindrical modes (i.e., $L(0,1), L(0,2)$ and $L(0,3))$ to an isotropic hollow tube model filled with viscous liquid (with given material properties). A pioneering proposal provided a combined estimation of both the cortical thickness and elastic coefficients from ex vivo measurements by minimizing the square differences between experimental and calculated frequencies using a least-square optimization criterion and a gradient-based method (i.e. built-in trust reflective region algorithm) [20]. The main limitation of that study was that the incomplete data were grouped into user-defined experimental trajectories, where each trajectory was associated to a specific Lamb branch. Nonetheless, this prior assignment is generally far from trivial, particularly in the case of in vivo measurements, where noise and soft tissue modes may corrupt the experimental trajectories. Consequently, an accurate optimization method for solving multiparametric inverse problems from multimode GWs is still lacking in the bone community.

Inverse problems based on GWs are, however, well accepted and widely used in the field of nondestructive testing (NDT) [21], including different applications such as the quality control of thin bonds or the characterization of composite materials. Among the 
few works dealing with higher-order guided modes, a number of studies was dedicated to the estimation of the elastic properties of isotropic plates [22, 23, 24], transverse isotropic materials as wood [25], or anisotropic structures [22, 26, 27]. Nonetheless, only a limited number of works proposed a combined estimation of both the structural and material properties. Karim et al. [22] used an inversion scheme based on the simplex algorithm to estimate the thickness and the elastic properties of an adhesive layer between two aluminum plates. Dean et al. [23] determined the thickness and two independent elastic constants of thin aluminum plates using a downhill simplex algorithm. Yan et al. [24] presented a particle swarm-based-simulated annealing optimization technique for retrieving the thickness and elastic properties of thin aluminum plates. However, all these approaches were applied to laboratory-controlled measurements on isotropic plates [22, 23], or even on simulated data [24].

In vivo data measured on cortical bone are, however, particularly complicated to deal with. Indeed, they are usually incomplete, in that some modes show up as piecewise curves as some frequency regions are not observed, and contaminated by noise or additional guided modes due to the overlying soft tissue [28, 29]. In addition, modal superposition cannot be avoided in regions of the frequency/wave number domain where the modes are close to each other, especially for short receivers array [30]. Thus, these ambiguities prevent to clearly identify the theoretical guided modes corresponding to the experimental data, especially for higher-order modes. As a consequence, typical approaches based on curve fitting, in which modes numbering prior to the inversion is required, do not serve our purpose, and a method in which the mode-order is kept blind is clearly needed $[24,30]$.

In this way, this work presents a global search approach, which enables us to estimate the thickness and a surrogate for the elastic properties (i.e., porosity) of cortical bone, avoiding any prior knowledge on the experimental data. This can be achieved by including an additional model parameter, in terms of a pairing vector, which represents the combination (i.e., number and position) of theoretical guided modes that are necessary to fit the experimental data. Genetic algorithms (GAs), which were already successfully applied for inverting GWs measurements in the NDT field [31, 32, 27] and geophysics [33], are used as search algorithm in the optimization, due to their capability of dealing with complex multidimensional and multimodal inverse problems. Our proposal is first validated on a series of laboratory-controlled materials (both isotropic and transverse isotropic) and then evaluated ex vivo on human radius specimens and in vivo on a cohort of healthy subjects. As a by-product of this study, it is worth pointing out that, despite the potential of both tools, proposals that exploit GAs coupled with multimode GWs data for identifying the thickness and porosity of cortical bone are, to the authors' best knowledge, nonexistent in the open literature.

The remaining of this paper is organized as follows. The experimental set-up and extraction of the dispersion curves are introduced in Section 2. The forward calculation of the theoretical dispersion curves is described in Section 3. The genetic algorithms-based identification framework is presented in Section 4. Finally, the results 
and discussion are exposed in Sections 5 and 6, respectively.

\section{Experimental measurements}

\subsection{Samples and reference measurements}

The proposed algorithm was first tested on laboratory-controlled measurements performed on a series of isotropic plates (aluminum, copper and brass) and composite plates/tube of different thickness. The composite material, given as transverse isotropic, is a bone-mimicking material made of short glass fibers embedded in an epoxy matrix (Sawbones ${ }^{\circledR}$, Pacific Research Laboratories Inc., Vashon Island, WA). The reference thickness of each plate/tube was directly derived from caliper measurements, repeated five times in the area of AT measurements. For the isotropic plates, reference values of the bulk wave velocities were taken from the literature [34]. For the bone-mimicking plates/tube, reference values of the stiffness coefficients, measured by resonant ultrasound spectroscopy, were available for the thicker plate [35].

Our approach was then evaluated on ex vivo measurements performed on three human radius specimens, which were supplied by the Département Universitaire d'Anatomie Rockefeller (Lyon, France) through the French program on voluntary corpse donation to science. These specimens were excised from fresh elderly cadavers (two males and one female, aged from 57 to 77 years old) and overlying soft tissues were removed. The donors furnished informed written consent to provide their tissues for investigation in accordance with legal clauses stated in the French Code of public health. The samples were kept frozen before use and lightly warmed to room temperature before acquiring the US data. In vivo measurements were also achieved in three healthy subjects (3 males aged from 27 to 34 years old) to test the robustness of our approach. They were all volunteers recruited from the laboratory staff. Note that informed consent was obtained from all subjects in accordance with regulations.

Measurements were performed on a predefined region of interest (ROI) at the proximal radius by positioning the center of the probe approximately $70 \mathrm{~mm}$ away from the radial styloid. The length of the ROI, prescribed by the length of the receiver array, was $20 \mathrm{~mm}$ on the postero-lateral face of the radius/forearm, as depicted in Figure 1. On that figure, $x_{1}$ and $x_{3}$-axes are supposed to be aligned with the direction of the principal symmetry of the material. The $x_{1} x_{2}$-plane is the isotropy plane, whereas the $x_{3}$-axis is oriented along the main bone axis. In the same way, for the bone-mimicking samples, the $x_{3}$-axis is oriented in the fibers' direction and the $x_{1}$-axis is normal to it.

Reference measurements were performed independently of the GWs measurements to assess the correctness of the US-based estimates. For the ex vivo samples, the cortical thickness and porosity were quantified in site-matched region using X-ray microcomputed tomography $(\mu \mathrm{CT})$ measurements with an isotropic voxel size of $9 \mu \mathrm{m}$ using a desktop $\mu \mathrm{CT}$ system (Bruker 1176, NV, Kontich, Belgium). For each specimen, a stack of 1200 sections was reconstructed (NRecon, Skyscan NV, Kontich, Belgium). The 

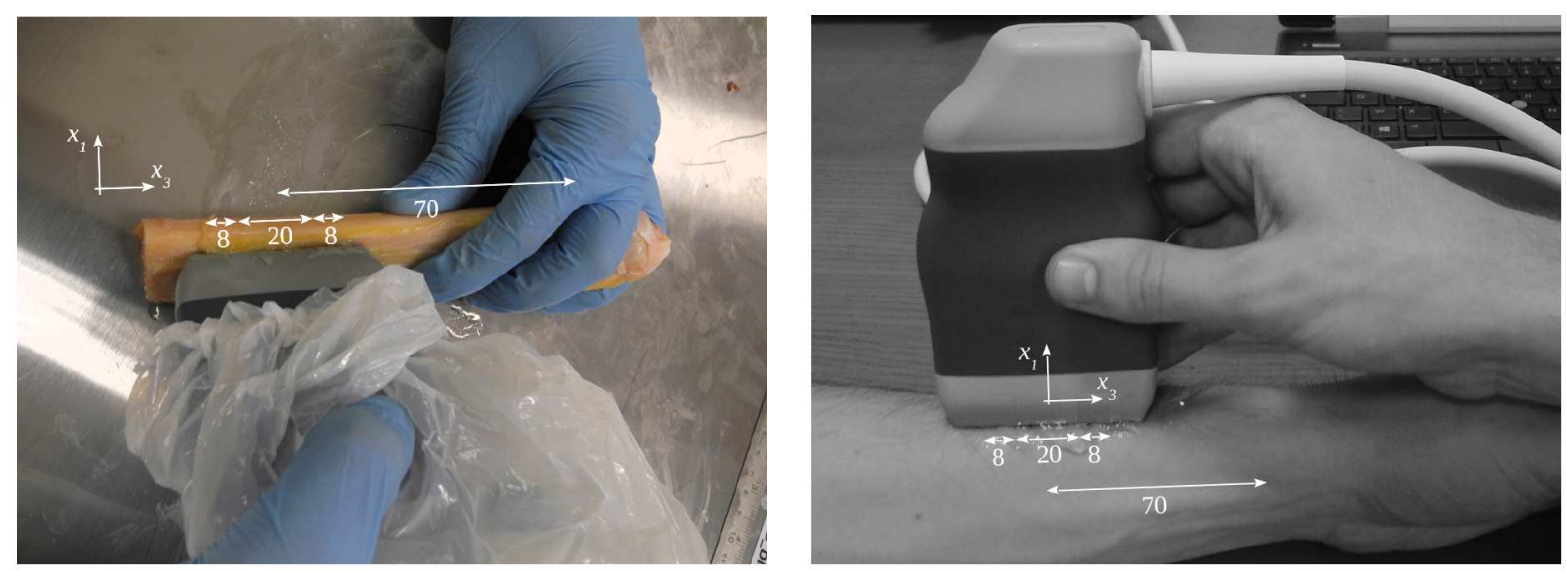

Figure 1. Measurement configuration with the multi-channel probe aligned along the main axis of bone: ex vivo (left) and in vivo (right). Distances are given in milimeters.

volumes of interest were selected for morphometric analysis using a semi-automated method (CTAnalyser, Skyscan NV, Kontich, Belgium). The external borders were contoured by the operator and then automatically interpolated over the longitudinal axis defining the volume of interest. Bone tissue and pore volumes were segmented using an adaptive threshold. Porosity was quantified as the ratio of the void volume over the total volume. Cortical thickness was assessed after global thresholding to encompass bone and pore by determining the average of the local thickness at each voxel-representing solid [36].

In vivo reference values of the cortical thickness were delivered by site-matched high resolution X-ray peripheral computed tomography (HR-pQCT) measurements (XtremCT, Scanco Medical, Bruttisellen, Switzerland) with a voxel size of $82 \mu \mathrm{m}$. The processing of these measurements was performed according to our previous study [20]. Although HR-pQCT has become a useful research tool for analyzing cortical bone structure in vivo, both the rather poor signal-to-noise ratio of the images and the limited spatial resolution (i.e., $128 \mu \mathrm{m}$ ) prevent reliable estimates of cortical porosity. Consequently, our in vivo estimates of porosity could not be confronted to an independent measurement.

Figure 2 depicts an example of typical transverse bone cross-sections obtained ex vivo and in vivo, along with the ROI used to compute the reference cortical thickness or/and porosity.

\subsection{Experimental set-up and extraction of the dispersion curves}

AT measurements were performed with a custom-made probe (Vermon, Tours, France), which consists of a 24 elements receiving array surrounded by two arrays of 5 emitters each. The three arrays of piezocomposite elements, aligned along the $x_{3}$-axis, were in contact with the samples and ultrasonic gel was used for coupling. The array pitch is $0.8 \mathrm{~mm}$ and the dimensions of each rectangular emitter are $0.8 \times 8 \mathrm{~mm}^{2}$. A distance 

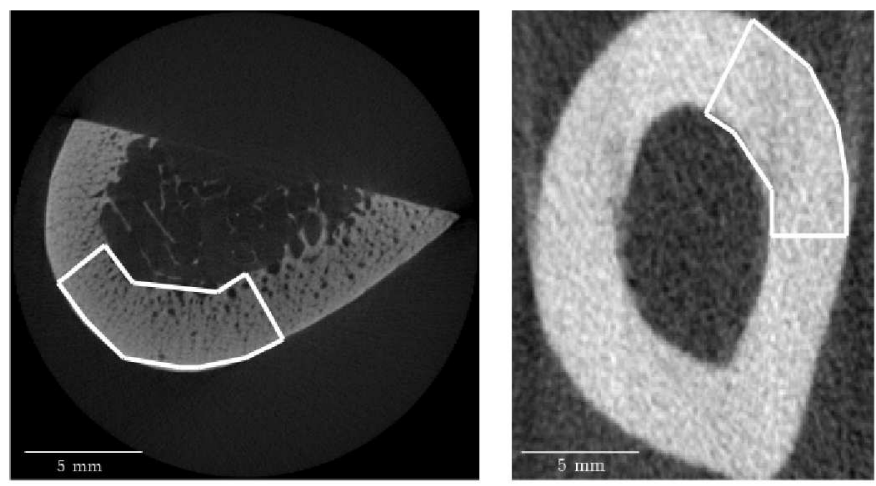

Figure 2. Example of transverse bone cross-sections obtained from (a) $\mu C T$ measurements of an ex vivo radius $(9 \mu \mathrm{m})$ and (b) HR-pQCT measurements on an in vivo forearm $(81 \mu \mathrm{m})$. The white area indicate the ROI used for determining the cortical thickness or/and porosity.

of $8 \mathrm{~mm}$ separates the receiving array from each emitting array (see Figure 1). This configuration allows the propagation of GWs in two opposite directions, which, for the in vivo measurements, enables the correction of the bias induced by the inclination angle between the probe and the bone, which could result from the presence of uneven overlying soft tissue in the measurements region [37].

A custom-made electronic device was used to transmit wideband ultrasonic pulses at a central frequency of $1 \mathrm{MHz}(-6 \mathrm{~dB}$ power spectrum spanning the frequency range from 0.4 to $1.6 \mathrm{MHz}$ ) and to record the received signals. An ensemble of 120 radiofrequency $(\mathrm{RF})$ signals corresponding to all possible pairs of emitter/receiver elements were digitized (12 bits, $20 \mathrm{MHz}, 1024$ samples) for each propagation direction after 16 averages by hardware (Althaïs Technologies, Tours, France).

The experimental dispersion curves, representing the frequency-dependent wave numbers (i.e., $k(f))$ of guided modes propagating in the waveguide, were extracted according to the basis introduced in [38]. Briefly, (1) for each propagation direction, the $120 \mathrm{RF}$ signals were Fourier transformed with respect to time and stored in a response matrix; (2) a singular value decomposition (SVD) was applied to the response matrix at each frequency; (3) enhancement of signal-to-noise ratio (SNR) was achieved by removing the singular vectors corresponding to the lowest singular values; (4) the projection of a testing vector (i.e., an attenuated spatial plane wave with a complex wave number [39]) onto the singular vector basis yielded the so-called Norm function, whose maxima correspond to the wave numbers of the guided modes; (5) the bidirectional correction, combining the data acquired from the two emitting arrays, was applied to the measurements according to $[37] ;(6)$ the $(f, k)$-pairs were extracted from the corrected Norm function using a dilation operator, which allowed a threshold-independent detection of the Norm function maxima; (7) outliers were removed from those pairs by applying statistical denoising over 10 measurement repetitions taken successively without moving the probe; and (8) the resulting denoised data on each direction were grouped together, leading to a single set of dispersion curves for each sample. The 
reader is referred to our previous reports $[38,39]$ for further details.

Figure 3 depicts examples of the $(f, k)$-pairs obtained after applying the aforementioned signal processing steps for both academic and bone samples.

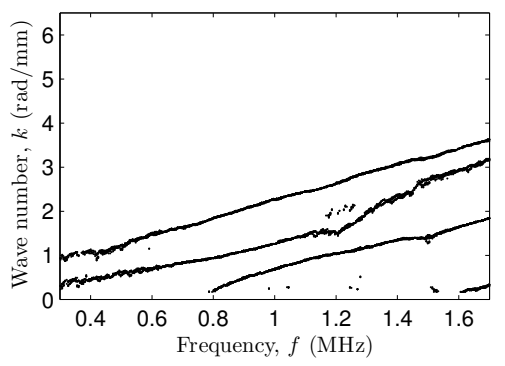

(a) Copper

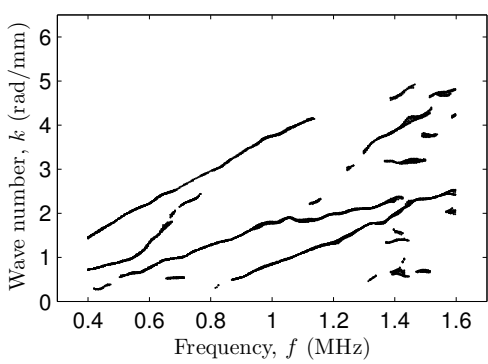

(b) Ex vivo

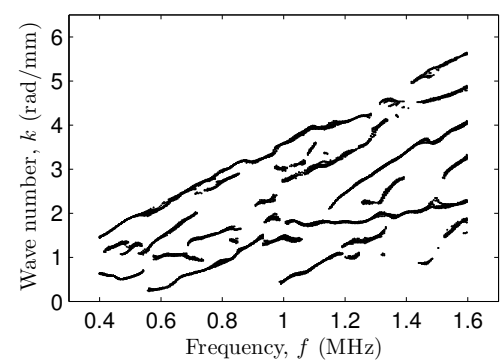

(b) In vivo

Figure 3. Example of the $(f, k)$-pairs obtained after applying the signal processing steps on the raw RF signals: (a) copper plate, (b) ex vivo specimen, and (c) in vivo subject.

\section{Forward calculation of the Lamb modes}

In the present study, a two-dimensional (2-D) transverse isotropic non absorbing free plate waveguide model is used to fit the experimental dispersion curves. This choice is motivated by our previous studies, which showed that the propagation of guided waves into tubular-shaped samples could be explained by a 2-D free plate model [40] and that a 2-D transverse isotropic free plate model, despite its simplicity, provided reliable cortical thickness estimates [20].

For such a model, the solutions of the corresponding Lamb wave equation for propagation in the meridian plane can be expressed as guided modes in the $f-k$ plane, which are determined by prescribed thickness $h$ of the waveguide, mass density $\rho$, and stiffness coefficients $c_{11}, c_{33}, c_{13}$, and $c_{55}$ [41]. Alternatively, the dispersion equation can be formulated as function of the bulk velocities, the mass density being embedded in the velocity parameters. The resulting model parameters that account for the stiffness of the waveguide are compound of two bulk wave velocities and two stiffness ratios, defined as:

$$
V_{L_{\perp}}=\sqrt{\frac{c_{11}}{\rho}}, V_{T}=\sqrt{\frac{c_{55}}{\rho}}, \frac{c_{13}}{c_{11}}, A R=\frac{c_{33}}{c_{11}}=\left(\frac{V_{L_{\|}}}{V_{L_{\perp}}}\right)^{2}
$$

where $V_{L_{\|}}, V_{L_{\perp}}$, and $V_{T}$ denote the longitudinal bulk wave velocities (along and normal to the $x_{3}$-axis) and the transverse bulk velocity, respectively.

According to the complexity of the tested material, three different sets of stiffness parameters were used in this study. For isotropic plates, only two independent model parameters are necessary to characterize the stiffness of the waveguide, namely $V_{L} \equiv V_{L_{\|}}=V_{L_{\perp}}$ and $V_{T}$ (i.e., the anisotropic ratio turns out to be constant, $A R=1$, and the stiffness ratio $c_{13} / c_{11}=1-2\left(V_{T} / V_{L}\right)^{2}$ depends on the two former bulk wave 
velocities). For transverse isotropic plates/tube, the four model parameters described in Equation (1), i.e., $\left\{c_{13} / c_{11}, A R, V_{L_{\perp}}, V_{T}\right\}$, are required.

To match the requirements of site-matched estimates of the cortical porosity measured by $\mu \mathrm{CT}$, the stiffness characterization of cortical bone is subjected to a further simplification. Hence, we assumed that the mesoscopic stiffness coefficients can be properly predicted by a single parameter [42], namely the cortical porosity $p$. The latter characterizes the volume fraction of pores into the material structure. To this end, a model of cortical bone mesoscopic elasticity based on asymptotic homogenization was used (source code available online [43]). The theory was described in details in the case of matrix isotropy in Parnell and Grimal [44], and extended later to the case of a transverse isotropic matrix [45]. In this model, cortical bone is represented as a two-phase composite material made of a homogeneous transversely isotropic matrix pervaded by periodically distributed water-filled cylindrical pores. This representation leads to transversely isotropic elasticity at the mesoscale. Given an elastic tensor $C^{m}$ describing the matrix elasticity, the mass density of the matrix $\rho^{m}$, an elastic tensor $C^{f}$ describing the elasticity of the material within the pores, and the volume fraction of pores $p$, a homogenized elastic tensor $C$ at the mesoscale and the apparent mass density $\rho$ can be calculated. In this study, we assume a universal matrix with fixed elastic tensor $C^{m}$ and density $\rho^{m}$ (set according to [43]), namely that the matrix is spatially homogeneous and uniform among individuals. In other words, one particular porosity $p$ corresponds to four effective elastic coefficients $c_{i j}$ plus one value of bone density $\rho$, which can, in turn, be written in terms of two stiffness ratios (i.e., $c_{13} / c_{11}$ and $\mathrm{AR}$ ) and two bulk wave velocities (i.e., $V_{L_{\perp}}$ and $\left.V_{T}\right)$ ) for computing the Lamb modes. Consequently, the model parametrization in terms of porosity does not change the dispersion equation, but can simply be seen as a sampling of the complete elasticity domain spanned by these four elastic parameters.

To illustrate the effect of the model parameters on the waveguide model, Figure 4 shows typical Lamb modes obtained for different thickness-porosity regimes, namely (a) $h=1.25 \mathrm{~mm}, p=20 \%$ and (b) $h=3 \mathrm{~mm}, p=5 \%$. As can be observed, the number of modes increases with increasing thickness, while the porosity yields a slope change of the modes (especially visible for low-order modes).

\section{Genetic algorithms-based identification framework}

Measurements of GWs, along with suitable waveguide modeling, have the potential to yield estimates of waveguide properties such as thickness and stiffness. Such a modelbased approach requires solving a multiparametric inverse problem (IP) for pairing the incomplete experimental data with Lamb modes. One of the most common approach to retrieve those estimates is to pose the IP in an optimization form. This section describes the objective function employed to compute the discrepancy between the measured and numerically predicted dispersion curves and introduce the underlying optimization algorithm used for solving the IP. 


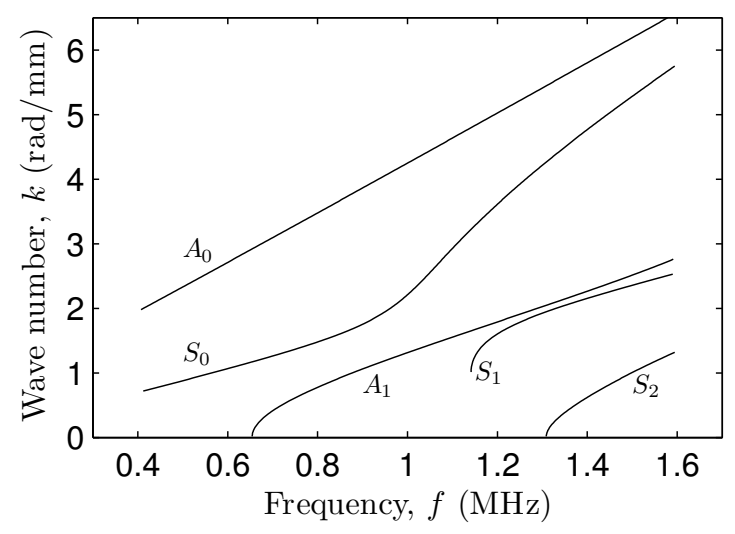

(a) $h=1.25 \mathrm{~mm}, p=20 \%$

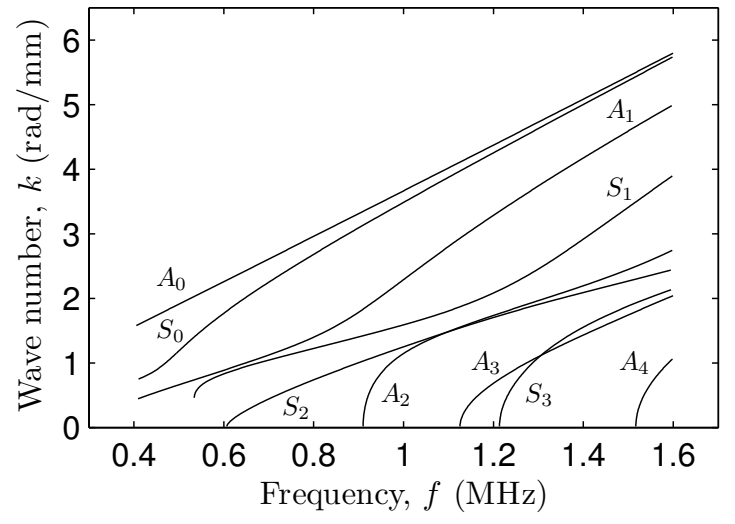

(b) $h=3 \mathrm{~mm}, p=5 \%$

Figure 4. Example of a 2-D transverse isotropic free plate model for (a) $h=1.25 \mathrm{~mm}, p=20 \%$ and (b) $h=3 m m, p=5 \%$.

\subsection{Objective function}

The difference between the experimental dispersion curves and Lamb modes is usually the most important constituent part of the objective function, in which the inversion process can be regarded as curve fitting (i.e., euclidean distance in a least-square sense). However, for in vivo multimode dispersion curves, it is far from trivial to a priori determine to which Lamb mode each data point of the experimental dispersion curves belongs, especially when dealing with a broad thickness range (e.g., $A_{1}$ in Figure 4a looks similar to $S_{2}$ in Figure $4 \mathrm{~b}$ ).

To overcome this difficulty, an additional model parameter is introduced here, which accounts for the pairing of the Lamb modes with the experimental data. This model parameter, denoted by $\boldsymbol{M}$, is a pairing vector that represents the combination (i.e., number and position) of Lamb modes that are necessary to explain the experimental data. In this way, the mode-order is kept blind and there is no need to identify the modes prior to solving the IP. The discrete bank of pairing vectors $\boldsymbol{M}$ is built following a combinatorial analysis based on three conditions: (1) There is at most $M^{\max }$ Lamb modes; (2) at most three modes can miss between two consecutive modes; and (3) within each pairing vector, the modes are sorted in ascending order according to the value of their cut-off frequency. The second condition is consistent with the fact that some modes are not excited (due to insufficient out-of-plane displacement and to high attenuation [20]) or pairwise higher-order modes (e.g., an anti-symmetric mode $A_{i}$ and its symmetric counterpart $S_{i}$ ) cannot be easily distinguished experimentally (e.g., $A_{3}$ and $S_{3}$ overlap in Figure $4 \mathrm{~b}$ ).

The resulting model parameters $\boldsymbol{\theta}=\left[\begin{array}{lll}h & \boldsymbol{S} & \boldsymbol{M}\end{array}\right]$, where $h$ denotes the cortical thickness and $\boldsymbol{S}$ is one of the three different sets of stiffness parameters described in Section 3 , are identified by a global search algorithm. In the present work, the objective 
function $F(\boldsymbol{\theta})$ consists of maximizing the occupancy rate of the Lamb modes. Hence,

$$
F(\boldsymbol{\theta})=\frac{1}{N} \sum_{i=1}^{M^{\max }} \frac{N_{i}^{\exp }}{N_{i}^{\text {th }}(\boldsymbol{\theta})} \quad\left(\boldsymbol{\theta}_{\text {inf }}<\boldsymbol{\theta}<\boldsymbol{\theta}_{\text {sup }}\right)
$$

restricted to:

$$
N_{i}^{\exp }= \begin{cases}N_{i}^{\text {in }} & \text { if } \quad N_{i}^{\exp }>0.1 \cdot \bar{N}^{\exp } \\ 0 & \text { otherwise }\end{cases}
$$

The variables $N_{i}^{\text {exp }}$ and $N_{i}^{\text {th }}(\boldsymbol{\theta})$ denote the number of experimental and theoretical data of a mode $i$, respectively; $\bar{N}^{\exp }$ is the mean of the $N_{i}^{\exp } ; N_{i}^{\text {in }}$ is the number of inliers of a mode $i$; and $N$ is the total number of experimental data. Basically, Equations (2)-(3) mean that experimental data can only form an experimental trajectory if a sufficiently large amount of them belong to a Lamb mode. Note that an experimental data is considered as an inlier of a mode $i$ if its euclidean distance $d$ to that mode satisfies the following condition:

$$
d=\sqrt{\left(\frac{f-f(\boldsymbol{\theta})}{f_{\max }}\right)^{2}+\left(\frac{k-k(\boldsymbol{\theta})}{k_{\max }}\right)^{2}} \leq d_{0}
$$

where $d_{0}=0.025$ is a user-defined dimensionless threshold, which approximately corresponds to the normalized resolution in $k$ (that is, $(\pi / L) / k_{\max }$ with $L$ being the length of the receivers array) [38, 39].

Finally, the optimal model parameters $\hat{\boldsymbol{\theta}}$ result from,

$$
\hat{\boldsymbol{\theta}}=\arg \max _{\boldsymbol{\theta}_{\text {inf }}<\boldsymbol{\theta}<\boldsymbol{\theta}_{\text {sup }}} F(\boldsymbol{\theta})
$$

where $\boldsymbol{\theta}_{\text {inf }}$ and $\boldsymbol{\theta}_{\text {sup }}$ denote the lower and upper bounds of the model parameters $\boldsymbol{\theta}$. Genetic algorithms are applied to maximize Equation (5), due to their capability of finding a near global solution in situations where the objective function is multidimensional and non-convex (i.e., has more than one local maximum).

\subsection{Genetic algorithms}

Genetic algorithms (GAs) are an heuristic optimization technique based on the rules of natural selection and genetics, which simulates the mechanisms of survival competition [46]. They have several advantages over gradient-based optimization techniques [47]: (1) they are robust, conceptually simple and can be used in situations where the objective function $F(\boldsymbol{\theta})$ is complex (e.g., multidimensional and non-convex); (2) they succeed in finding a nearly global IP solution without the need of an accurate initial guess for the model parameters $\boldsymbol{\theta}$ (this is of particular interest for bone, where the stiffness coefficients are generally unknown); and (3) the search mechanisms possess an inherent parallelism that allows a rapid sampling of the solution space.

In contrast to gradient-based optimization techniques, GAs do not update a single solution at a generic iteration on both the basis of the modeling error and gradient values. The basic idea of GAs is, instead, to select among a population of individuals 
that represent a set of potential IP solutions, the individual that yields the optimal solution [27]. Figure 5 depicts the flowchart of the model-based IP approach using GAs.

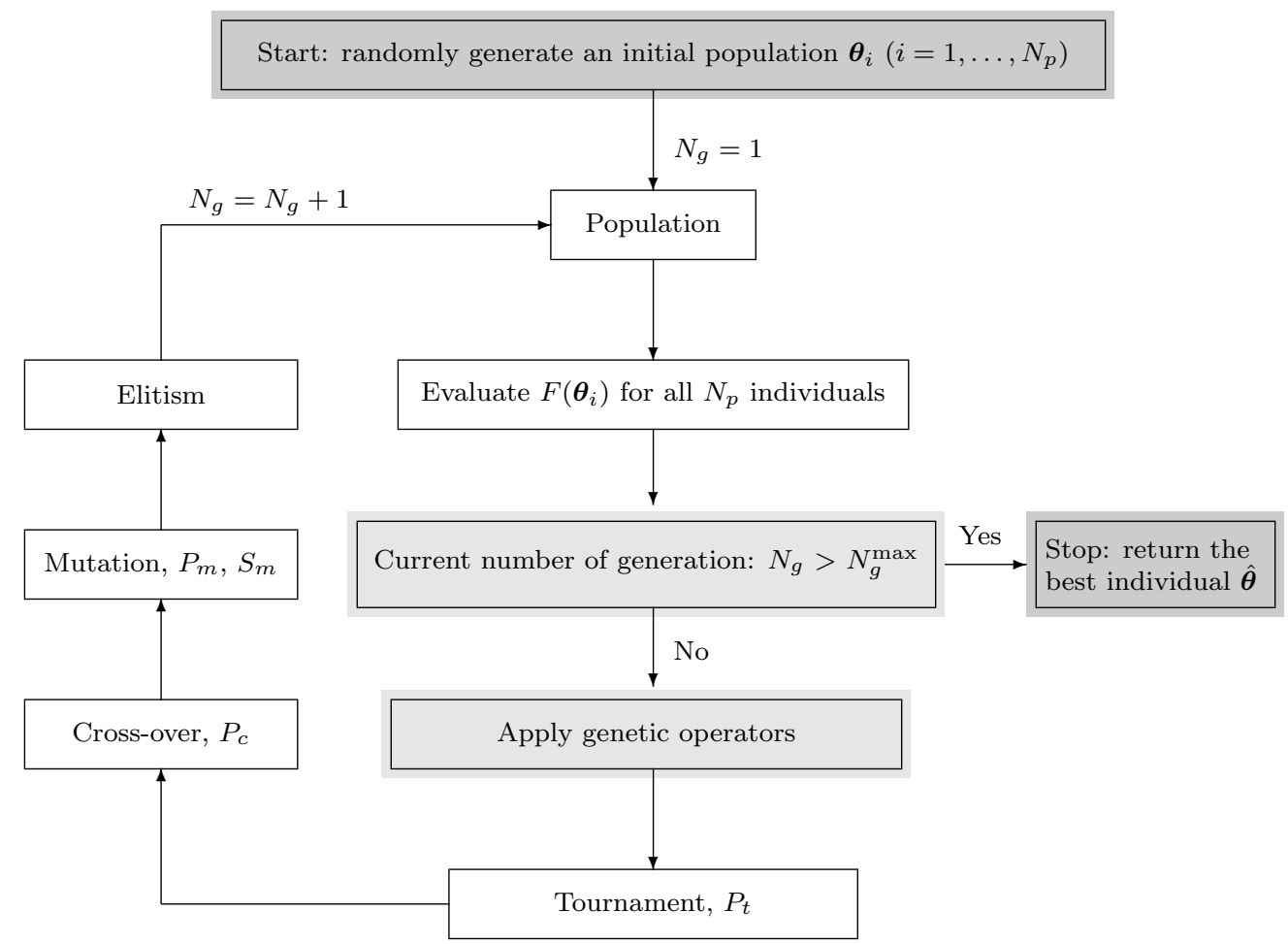

Figure 5. Flowchart of the model-based IP approach using GAs. User-defined variables: $N_{p}$ : Number of individuals per generation; $N_{g}^{\max }$ : Number of generations; $P_{t}$ : Probability of tournament; $P_{c}$ : Probability of crossover; $P_{m}$ : Probability of mutation; and $S_{m}$ : Scale of mutation.

The underlying steps of GAs are described in more detail in the following:

(i) An initial population of $N_{p}$ individuals (called chromosomes) is randomly generated. The population comprises a group of chromosomes that represent potential solutions $\boldsymbol{\theta}_{i},\left(i=1, \ldots, N_{p}\right)$, in the solution space spanned by $\boldsymbol{\theta}_{\text {inf }}<\boldsymbol{\theta}<\boldsymbol{\theta}_{\text {sup }}$ (see Table 1 below).

(ii) Each solution $\boldsymbol{\theta}_{i}$ is evaluated by computing the objective function $F\left(\boldsymbol{\theta}_{i}\right)$ described in Equation (3), for which one forward problem is solved independently.

(iii) The stopping criterion of the GAs scheme is checked. The algorithm stops if the maximal number of generations $N_{g}^{\max }$ is reached. If this criterion is satisfied, the best individual $\hat{\boldsymbol{\theta}}$ obtained is returned as the final IP solution.

(iv) If not, a new (child) population is formed by stochastically modifying the individuals (parents) of the previous generation, applying genetic operators such as tournament, crossover, mutation, and elitism to inject genetic diversity in the population (i.e., to ensure that the solution does not fall in local maxima). Tournaments, associated with a probability $P_{t}$, are first run among two individuals chosen at random from the population. The winners (parents) are selected for crossover. The crossover operation is then applied with a probability $P_{c}$ to produce new individuals (children) 
that have some parts of both parents' genetic characteristics. This operator makes the solution set jumps in the solution space. Secondly, mutation is randomly applied, with a probability $P_{m}$ and scale $S_{m}$, to modify elements in the parents' chromosomes, thus preventing the children from having only characteristics of the previous generation. Finally, elitism allows the best individuals from the current generation to carry over the next, thus guaranteeing that the solution will not decrease from one generation to the next. In this work, the best chromosome is carried to the next generation without modification.

(v) Using the individuals of the new generation, the algorithm restarts from step (ii) and the procedure continues until the aforementioned stopping criterion is met.

The choice of $\left\{N_{g}^{\max }, N_{p}\right\}$, along with the probabilities of the genetic operators $\left\{P_{t}=0.7, P_{c}=0.8, P_{m}=0.1, S_{m}=0.1\right\}$, are empirical variables set so that the convergence to a near global optimum is guaranteed, while establishing a trade-off between the IP error and the computational cost [48]. In this work, the convergence of the algorithm was trained over a group of experimental data by examining the evolution of the currently optimal solution by increasing $\left\{N_{g}^{\max }, N_{p}\right\}$. After a certain number of iterations, we could no longer see any further improvement of the solutions and the optimal variables were fixed when applying the algorithm to the remaining data.

\subsection{Settings and conditioning of the genetic algorithms}

The bounds of the model parameters $\boldsymbol{\theta}$ that define the problem domain were selected as follows: firstly, the thickness bounds of the plates/tube were chosen so that the corresponding interval covers all the values measured by caliper. Secondly, the bulk wave velocities bounds for the isotropic plates covered values encountered in the literature for all the materials tested here [34]. Thirdly, the bulk wave velocities and stiffness ratios range for the bone-mimicking plates/tube were defined as large as in [20]. Lastly, the bounds of the model parameters for cortical bone were taken according to physiological observations found in the literature [5]. The bounds of the model parameters $\boldsymbol{\theta}$ and the empirical variables of the GAs are summarized in Table 1 for all the samples tested in this work.

\begin{tabular}{|c|c|c|c|c|c|c|c|c|c|}
\hline & \multicolumn{7}{|c|}{ Bounds of the model parameters $\boldsymbol{\theta}:\left[\begin{array}{ll}\boldsymbol{\theta}_{\text {inf }} & \boldsymbol{\theta}_{\text {sup }}\end{array}\right]$} & \multicolumn{2}{|c|}{ GAs variables } \\
\hline & $\frac{c_{13}}{c_{11}}$ & $\begin{array}{c}A R \\
-\end{array}$ & $\begin{array}{c}V_{L_{\perp}} \\
(\mathrm{mm} / \mu \mathrm{s})\end{array}$ & $\begin{array}{c}V_{T} \\
(\mathrm{~mm} / \mu \mathrm{s})\end{array}$ & $\begin{array}{c}p \\
(\%)\end{array}$ & $\begin{array}{c}h \\
(\mathrm{~mm})\end{array}$ & $\begin{array}{c}M^{\max } \\
\quad-\end{array}$ & $N_{g}^{\max }$ & $N_{p}$ \\
\hline Isotropic plates & - & - & {$[3.767 .58]$} & {$[1.843 .71]$} & & & & 320 & 80 \\
\hline Bone-mimicking materials & {$\left[\begin{array}{ll}0.2 & 0.7\end{array}\right]$} & {$\left[\begin{array}{ll}1.1 & 2.5\end{array}\right]$} & {$[1.583 .74]$} & {$\left[\begin{array}{ll}1 & 2\end{array}\right]$} & [م- & {$\left[\begin{array}{ll}0.5 & 5.5\end{array}\right]$} & 14 & 400 & 100 \\
\hline Cortical bone & - & - & - & - & {$\left[\begin{array}{ll}0 & 30\end{array}\right]$} & {$[0.54]$} & 10 & 240 & 60 \\
\hline
\end{tabular}

Table 1. GAs settings.

Note also that the pairing vector $\boldsymbol{M}$ is naturally dependent on the cortical thickness $h$ (i.e., the maximal number of modes increases with increasing thickness, as can be seen in Figure 4). As a consequence, the populations in the GAs are built so that 
the model parameters $\boldsymbol{\theta}$ satisfy the relation between $h$ and $M^{\text {max }}$, depicted in Figure 6. In the same vein, for the isotropic case, the populations are built so that the

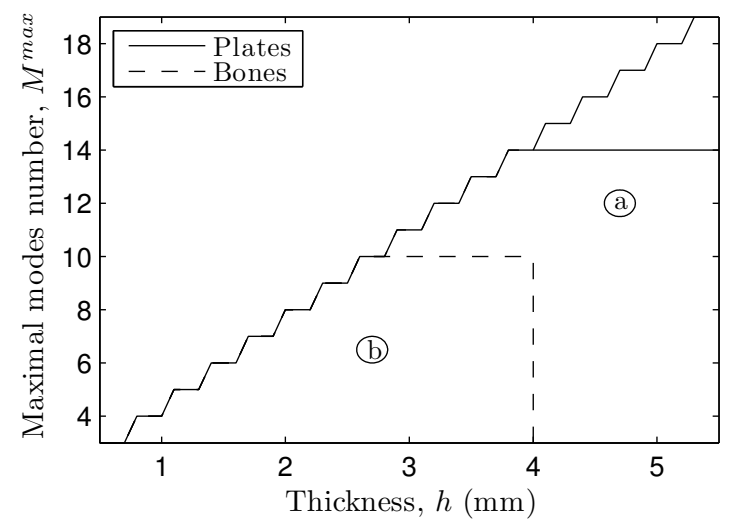

Figure 6. Relationship between the maximum Lamb modes number $M^{\text {max }}$ and the thickness $h:(a)+$ (b) depict the allowed region for academic plates/tube, whereas (b) depicts the allowed region for bone.

thermodynamic condition for isotropy is fulfilled [49], that is $V_{T}<V_{L} / \sqrt{2}$. Such a condition can unfortunately not be verified for the transverse isotropic case, because the stiffness coefficient $c_{66}$ is not available in a $2-\mathrm{D}$ waveguide model. In this way, this conditioning allows avoiding unphysical solutions in the populations, thus strengthening the convergence of the algorithm over the course of the generations.

\section{Results}

\subsection{Case study: Isotropic plates}

First, results on isotropic plates are presented. Figure 7 depicts the optimal matching between the experimental data and the Lamb modes. As can be observed, a remarkable agreement is found between the experimental data and the model. The US-based estimates $\hat{\boldsymbol{\theta}}$, which are compared to reference values, are summarized in Table 2. The

\begin{tabular}{|c|c|c|c|c|c|c|c|}
\hline & \multicolumn{4}{|c|}{ Optimal model parameters $\hat{\boldsymbol{\theta}}$} & \multicolumn{3}{|c|}{ Reference values } \\
\hline & \multirow{2}{*}{$\begin{array}{c}\text { Thickness } \\
\text { (mm) } \\
h\end{array}$} & \multicolumn{2}{|c|}{$\begin{array}{l}\text { Bulk velocities } \\
\quad(\mathrm{mm} / \mu \mathrm{s})\end{array}$} & \multirow{2}{*}{$\begin{array}{c}\text { Pairing vector } \\
-\end{array}$} & \multirow{2}{*}{$\begin{array}{c}\begin{array}{c}\text { Thickness } \\
(\mathrm{mm})\end{array} \\
h_{\mathrm{ref}} \\
\end{array}$} & \multicolumn{2}{|c|}{$\begin{array}{l}\text { Bulk velocities } \\
\qquad(\mathrm{mm} / \mu \mathrm{s})\end{array}$} \\
\hline & & $V_{L}$ & $V_{T}$ & & & $V_{L_{\text {ref }}}$ & $V_{T_{\text {ref }}}$ \\
\hline Aluminum & 2.09 & 6.79 & 3.23 & {$\left[\begin{array}{lllll}A_{0} & S_{0} & A_{1} & S_{1} & S_{2}\end{array}\right]$} & $2.02 \pm 0.02$ & 6.38 & 3.11 \\
\hline Copper & $\begin{array}{l}2.11 \\
5.12\end{array}$ & $\begin{array}{l}4.88 \\
4.87\end{array}$ & $\begin{array}{l}2.40 \\
2.34\end{array}$ & 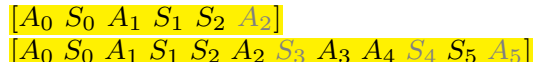 & $\begin{array}{l}2.03 \pm 0.02 \\
5.03 \pm 0.02\end{array}$ & 4.76 & 2.33 \\
\hline Brass & 1.51 & 4.52 & 2.22 & {$\left[\begin{array}{lllll}A_{0} & S_{0} & A_{1} & S_{1} & S_{2}\end{array}\right]$} & $1.48 \pm 0.02$ & 4.37 & 2.10 \\
\hline
\end{tabular}

Table 2. Isotropic plates: Reference values and US-based estimates of thickness and bulk velocities (modes that are missing in the optimal pairing vector are displayed in gray).

relative difference on thickness is lower than $4 \%$, while the relative differences on bulk velocities are lower than $6.5 \%$ and $6 \%$ for $V_{L}$ and $V_{T}$, respectively. Note that both the 


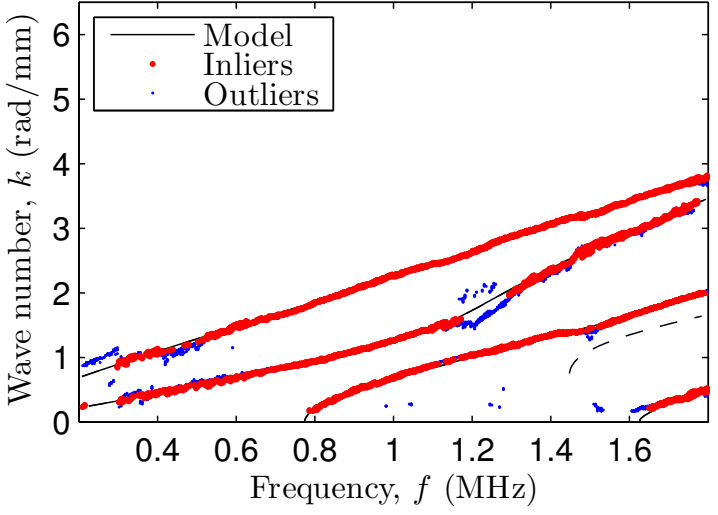

(a) 2-mm aluminum plate

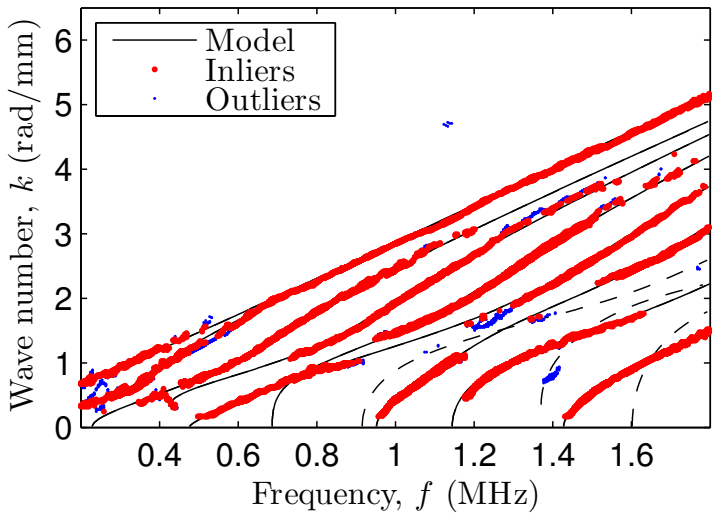

(c) 5-mm copper plate

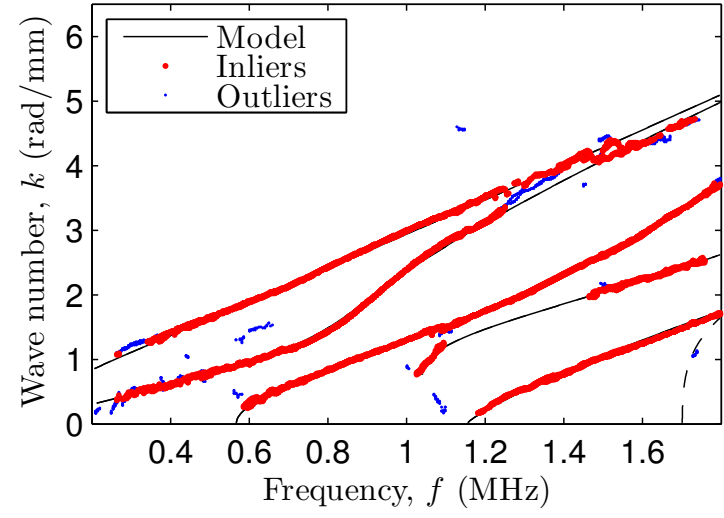

(b) 2-mm copper plate

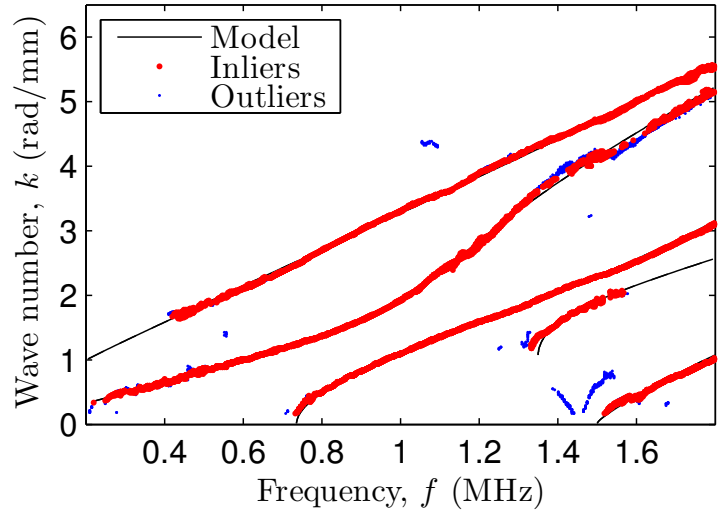

(d) 1.5-mm brass plate

Figure 7. Optimal matching between the experimental data (dots) and the Lamb modes (lines) for the isotropic plates: (a) 2-mm aluminum, (b) 2-mm copper, (c) 5-mm copper and (d) 1.5-mm brass. Inliers and outilers are displayed in red and blue dots, respectively. Modes that are missing in the optimal pairing vector $\boldsymbol{M}$ are displayed in discontinuous lines.

structural and material US-based estimates generally tend to slightly overestimate the reference values.

One strength of the proposed algorithm is to be able not only to retrieve reliable estimates of the plate thickness (i.e., $h$ ) and bulk velocities (i.e., $V_{L}, V_{T}$ ), but also to recognize Lamb modes that are not excited experimentally (discontinuous lines in Figure 7), thanks to the pairing vector (i.e., $\boldsymbol{M}$ ) that prevents possible mode misidentification. Note that both antisymmetric and symmetric modes can miss (indicated as light gray symbols in Table 2), depending on the material properties, the thickness regime and the frequency bandwidth.

\subsection{Case study: Bone-mimicking plates}

Second, results on bone-mimicking plates and tube are presented. Figure 8 depicts the optimal matching between the experimental data and the Lamb modes. Again, a good agreement is found between the experimental data and the model. Table 3 summarizes the US-based estimates $\hat{\boldsymbol{\theta}}$, which are compared to reference values. The US- 


\section{Page 15 of 24}

1

2

3

4

5

6

7

8

9

10

11

12

13

14

15

16

17

18

19

20

21

22

23

24

25

26

27

28

29

30

31

32

33

34

35

36

37

38

39

40

41

42

43

44

45

46

47

48

49

50

51

52

53

54

55

56

57

58

59

60

Preprint submitted to Physics in Medicine and Biology

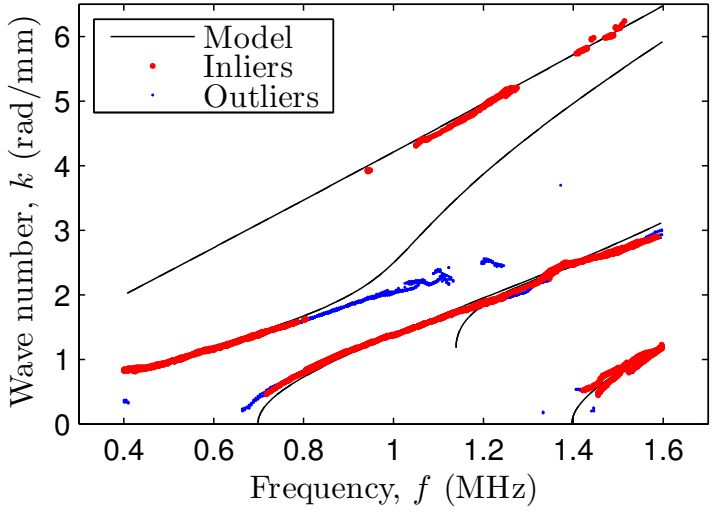

(a) 1-mm bone-mimicking plate

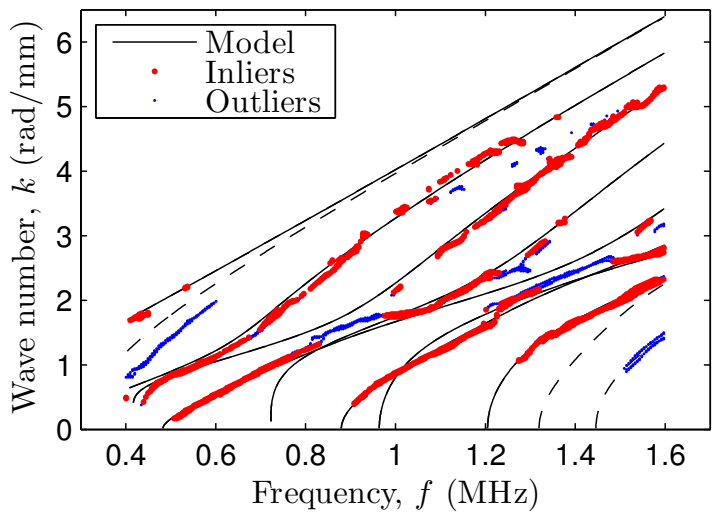

(c) 3-mm bone-mimicking plate

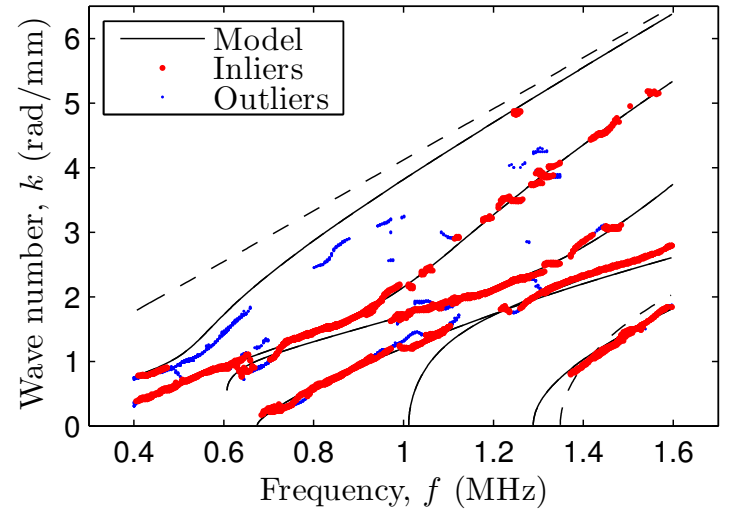

(b) 2-mm bone-mimicking plate

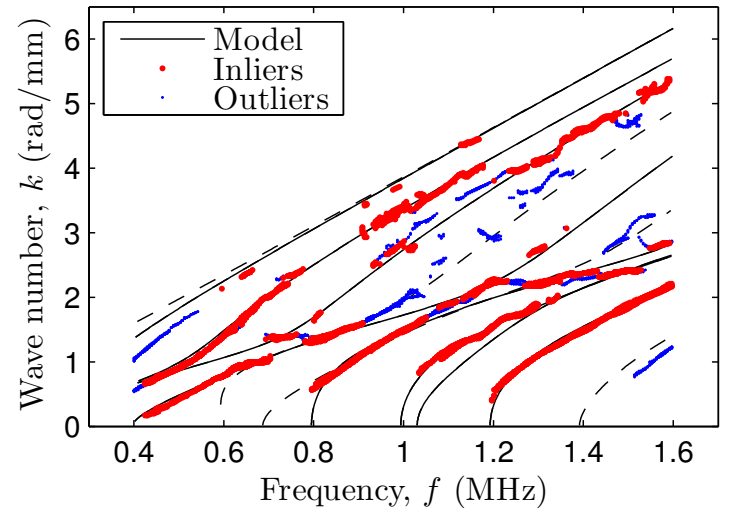

(d) 4-mm bone-mimicking plate

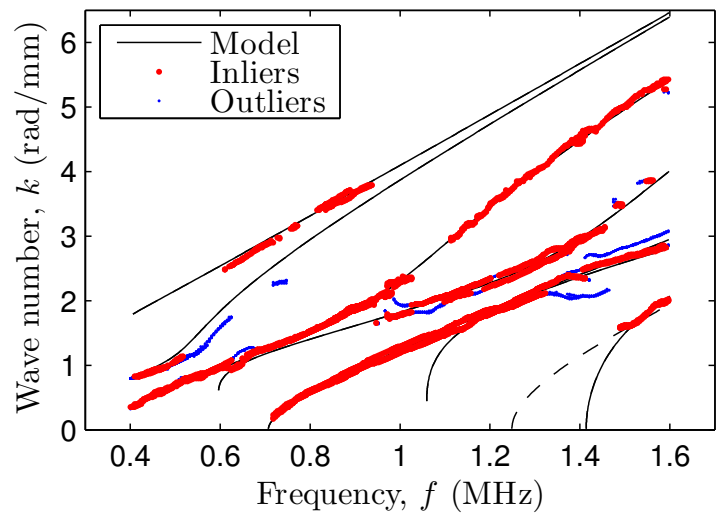

(e) 2-mm bone-mimicking tube

Figure 8. Optimal matching between the experimental data (dots) and the Lamb modes (continuous lines) for the bone-mimicking plates/tube. Inliers and outilers are displayed in red and blue dots, respectively. Modes that are missing in the optimal pairing vector $\boldsymbol{M}$ are displayed in discontinuous lines.

based approach delivers estimates of stiffness ratios and bulk wave velocities, while the reference method provides stiffness coefficients. Consequently, a face-to-face comparison can only be performed by making use of Equation (1) with a given mass density $\rho=1.64$ $\mathrm{g} / \mathrm{cm}^{3}$ (the resulting stiffness ratios and bulk wave velocities are marked in bold in the 
table). As can be observed, the relative difference on thickness is lower than $3 \%$, while the relative differences on bulk wave velocities are less than $7 \%$ for $V_{L_{\perp}}$ (around 200 $\mathrm{m} / \mathrm{s}$ ), less than $7 \%$ for $V_{T}$ (around $100 \mathrm{~m} / \mathrm{s}$ ), and less than $6 \%$ for $V_{L_{\|}}$(around $200 \mathrm{~m} / \mathrm{s}$ ). The inter-sample repeatability, calculated as the half-range divided by the median value over the five samples, was less than $4 \%$ for $V_{L_{\perp}}$ and $V_{T}$, and less than $5 \%$ for $V_{L_{\|}}$.

\begin{tabular}{|c|c|c|c|c|c|c|c|}
\hline & \multicolumn{6}{|c|}{ Optimal model parameters $\hat{\boldsymbol{\theta}}$} & \multirow{3}{*}{$\begin{array}{c}\begin{array}{c}\text { Reference } \\
\text { thickness } \\
(\mathrm{mm})\end{array} \\
h_{\mathrm{ref}}\end{array}$} \\
\hline & \multirow{2}{*}{$\begin{array}{c}\begin{array}{c}\text { Thickness } \\
(\mathrm{mm})\end{array} \\
h\end{array}$} & \multicolumn{2}{|c|}{$\begin{array}{c}\text { Stiffness ratios } \\
-\end{array}$} & \multicolumn{2}{|c|}{$\begin{array}{l}\text { Bulk velocities } \\
\qquad(\mathrm{mm} / \mu \mathrm{s})\end{array}$} & \multirow{2}{*}{$\begin{array}{c}\text { Pairing vector } \\
- \\
M\end{array}$} & \\
\hline & & $\frac{c_{13}}{c_{11}}$ & $A R$ & $V_{L_{\perp}}$ & $V_{T}$ & & \\
\hline Plate 1 & 1.21 & 0.45 & 1.43 & 2.88 & 1.70 & {$\left[\begin{array}{lllll}A_{0} & S_{0} & A_{1} & S_{1} & S_{2}\end{array}\right]$} & $1.25 \pm 0.02$ \\
\hline Plate 2 & 2.40 & 0.49 & 1.49 & 3.10 & 1.62 & {$\left[\begin{array}{llllllll}A_{0} & S_{0} & A_{1} & S_{1} & S_{2} & A_{2} & A_{3} & S_{3}\end{array}\right]$} & $2.34 \pm 0.05$ \\
\hline Plate 3 & 3.44 & 0.50 & 1.43 & 3.03 & 1.66 & {$\left[\begin{array}{lllllllllll}A_{0} & S_{0} & A_{1} & S_{1} & S_{2} & A_{2} & A_{3} & S_{3} & A_{4} & S_{4} & S_{5}\end{array}\right]$} & $3.48 \pm 0.21$ \\
\hline Plate 4 & 4.34 & 0.46 & 1.51 & 2.98 & 1.73 & {$\left[\begin{array}{llllllllllll}A_{0} & S_{0} & A_{1} & S_{1} & S_{2} & A_{2} & A_{3} & S_{3} & A_{4} & S_{4} & S_{5} & A_{5}\end{array}\right]$} & $4.21 \pm 0.11$ \\
\hline Tube & 2.33 & 0.46 & 1.46 & 2.91 & 1.65 & {$\left[\begin{array}{llllllll}A_{0} & S_{0} & A_{1} & S_{1} & S_{2} & A_{2} & A_{3} & S_{3}\end{array}\right]$} & $2.32 \pm 0.05$ \\
\hline Ref. $[\overline{35}]$ & -- & 0.50 & 1.50 & 2.91 & 1.62 & $\begin{array}{c}----------------- \\
-\end{array}$ & ------ \\
\hline
\end{tabular}

Table 3. Bone-mimicking samples: Reference values and US-based estimates of thickness, stiffness ratios and bulk velocities (modes that are missing in the optimal pairing vector are displayed in gray).

Note that measurements on the tube of circular cross-section (Figure 8e) do not significantly differ from those on the thickness-equivalent plate (Figure 8b). Consequently, these measurements do not affect the identification of the model parameters, thus strengthening the hypothesis that, in our case, measurements on a non-flat contact surface can conveniently be modeled with a 2-D free plate model [20, 40].

As in the isotropic case, the proposed algorithm is able to retrieve reliable estimates of the plate thickness, stiffness ratios and bulk wave velocities, and also to recognize Lamb modes that are missing experimentally (discontinuous lines). As for isotropic materials, it results that both antisymmetric and symmetric modes can miss.

\subsection{Cortical bone assessment}

Dispersion curves were measured on all the ex vivo radii and in vivo subjects. Figures 9 depict the experimental data along with the optimal model for the three ex vivo and in vivo cases, respectively.

The US-based estimates of cortical thickness and porosity, along with the reference values measured by site-matched X-ray-based techniques are summarized in Table 4.

Despite a lower SNR and the possible presence of additional guided modes (for the in vivo cases), which show through a larger number of outliers than for the academic materials, a reasonable agreement was found between the experimental data and the model. The absolute differences on cortical thickness were lower than $0.2 \mathrm{~mm}$ and are in the order of magnitude of the reference standard deviations. On the other hand, the absolute difference on the ex vivo porosity was around 1.5\%. US-based estimates of the porosity cannot be validated in vivo, but are coherent with the values estimated ex 


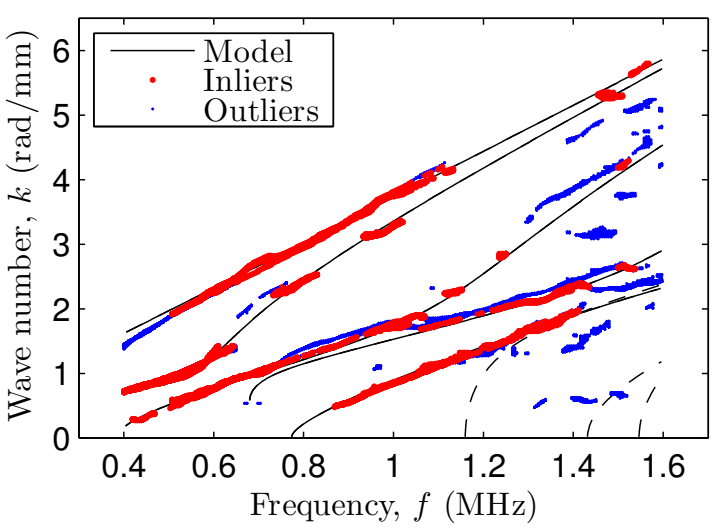

(a) Ex vivo 1

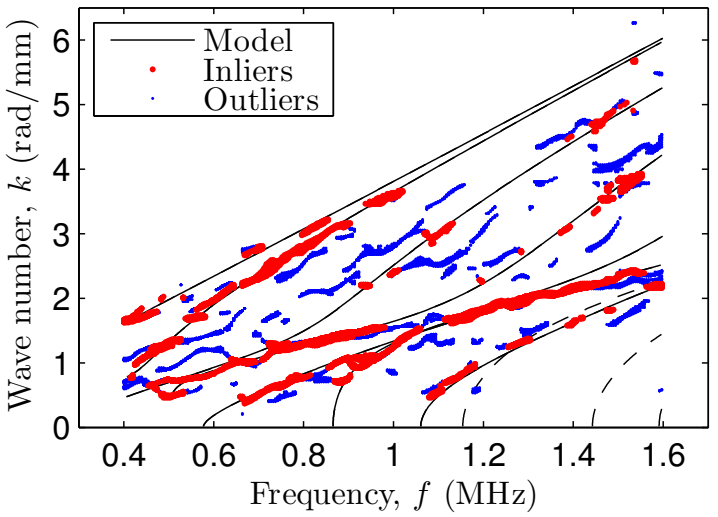

(b) Ex vivo 2

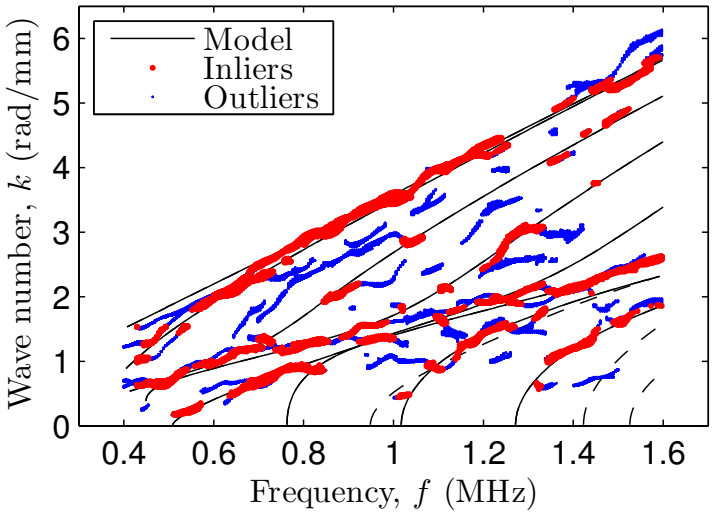

(c) Ex vivo 3

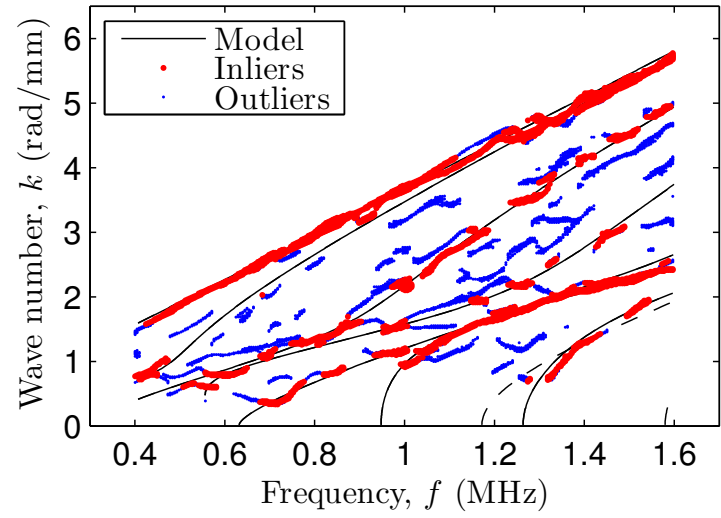

(d) In vivo 1

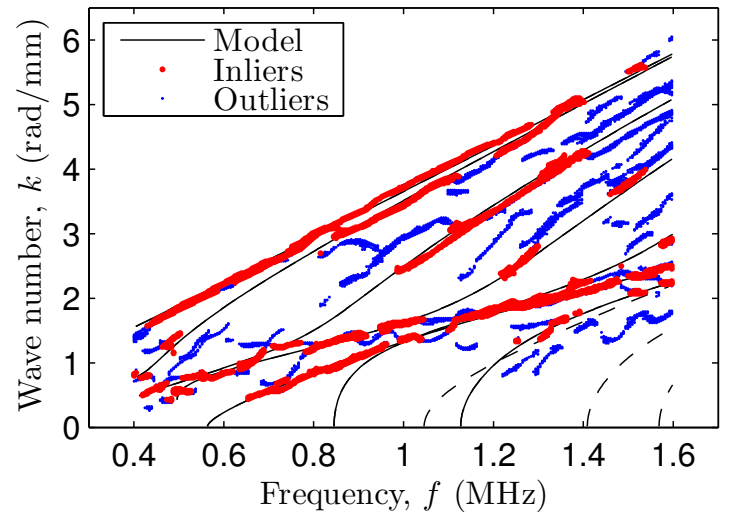

(e) In vivo 2

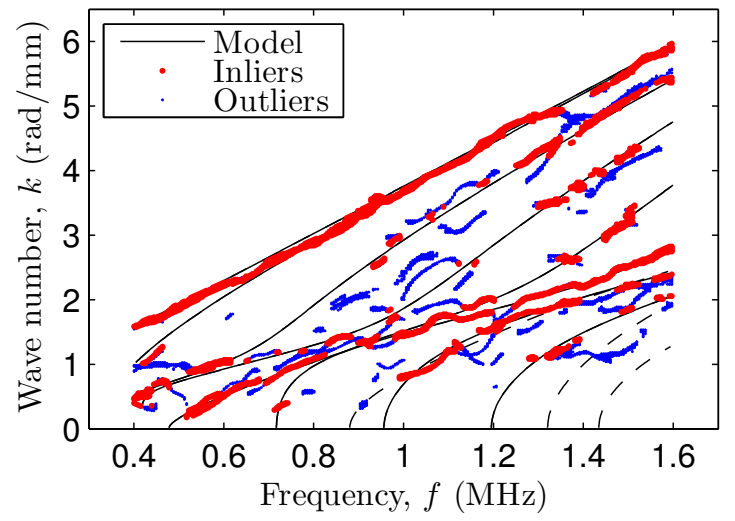

(f) In vivo 3

Figure 9. Optimal matching between experimental data (dots) and Lamb modes (continuous lines) for ex vivo radii $((a)-(c))$ and in vivo subjects $((d)-(f))$. Inliers and outilers are displayed in red and blue dots, respectively. Modes that are missing in the optimal pairing vector $\boldsymbol{M}$ are displayed in discontinuous lines.

vivo and with $\mu$ CT-estimates tabulated in the literature (e.g., [3 - 15]\% [50], [2-14]\% [51]). As in the previous cases, both antisymmetric and symmetric modes can miss in the data. It is worth pointing out, that for bones of similar thickness, there is no difference between the modes-order for ex vivo and in vivo samples, thus suggesting that 


\begin{tabular}{|c|c|c|c|c|c|c|}
\hline & & \multicolumn{3}{|c|}{ Optimal model parameters $\hat{\boldsymbol{\theta}}$} & \multicolumn{2}{|c|}{$\begin{array}{l}\text { Reference values } \\
\mu \mathrm{CT} / \mathrm{HR} \text {-pQCT }\end{array}$} \\
\hline & & $\begin{array}{l}\text { Thickness } \\
\text { (mm) }\end{array}$ & $\begin{array}{l}\text { Porosity } \\
(\%)\end{array}$ & $\begin{array}{c}\text { Pairing vector } \\
-\end{array}$ & $\begin{array}{l}\text { Thickness } \\
\text { (mm) }\end{array}$ & $\begin{array}{l}\text { Porosity } \\
(\%)\end{array}$ \\
\hline & & $h$ & $p$ & $M$ & $h_{\text {ref }}$ & $p_{\text {ref }}$ \\
\hline Ex vivo & 3 & 3.6 & 2.6 & {$\left[\begin{array}{lllllllllll}A_{0} & S_{0} & A_{1} & S_{1} & S_{2} & A_{2} & A_{3} & S_{3} & A_{4} & S_{4} & S_{5}\end{array}\right]$} & $3.8 \pm 0.1$ & $4.1 \pm 0.2$ \\
\hline \multirow{3}{*}{ In vivo } & 1 & 2.9 & 5.2 & {$\left[\begin{array}{lllllllll}A_{0} & S_{0} & A_{1} & S_{1} & S_{2} & A_{2} & A_{3} & S_{3} & A_{4}\end{array}\right]$} & $3.0 \pm 0.2$ & - \\
\hline & 2 & 3.2 & 5.0 & {$\left[\begin{array}{llllllllll}A_{0} & S_{0} & A_{1} & S_{1} & S_{2} & A_{2} & A_{3} & S_{3} & A_{4} & S_{4}\end{array}\right]$} & $3.3 \pm 0.2$ & - \\
\hline & 3 & 3.6 & 9.5 & 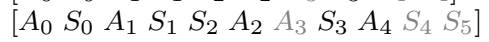 & $3.5 \pm 0.2$ & - \\
\hline
\end{tabular}

Table 4. Cortical bones: Reference values and US-based estimates of thickness and porosity (modes that are missing in the optimal pairing vector are displayed in gray).

the overlying soft tissue do not significantly affect the modes progagating in bone.

\section{Discussion}

In this study, we report on a GAs-based inversion method using multimode guided waves for a combined identification of cortical thickness and porosity of bone samples. A strength of our proposal is that the global search performed by GAs, along with the definition of an objective function in terms of the occupancy rate, do not require any prior knowledge neither on the model parameters (e.g., initial guess as in conventional gradient-based approaches) nor on the way to associate experimental data to a specific Lamb mode (i.e., the mode-order is kept blind through the introduction of a pairing vector).

The method was first validated on a series of laboratory-controlled measurements performed on isotropic plates and bone-mimicking phantoms, and then evaluated on a few ex vivo and in vivo human radii. Correct estimates could be retrieved through the multiparametric inversion, even when higher-order modes are superimposed in the experimental dispersion curves or when it is not trivial to a priori determine which Lamb mode each data point of the experimental dispersion curve belongs to. The optimization procedure allowing the estimation of the model parameters is (1) userindependent and takes into account all data, unlike our former studies [20, 40], where strong prior knowledge was necessary to fit the experimental trajectories to the Lamb modes; and (2) not restricted to a specific number of model parameters, where an exhaustive search might prove rather intractable from a computational viewpoint (our inversion scheme, solved and parallelized on a 24-cores desktop computer - Intel Xeon CPU E5-2620 v2 @ 2.10GHz -, takes around 10 minutes for a single data set).

Although multiparametric inversion methods using multimode GWs have been widely used for the NDE of different waveguide structures, this work introduces for the first time a pairing vector to reduce the ambiguity for identifying the modes and gathers the complexity of aspects that were usually studied independently, namely: (1) measurements on both academic and singular waveguide structures; (2) the use of 
a global search algorithm in the inversion procedure; and (3) the estimation of both structural and material properties. To the authors' best knowledge, the excitability of guided modes has not been properly described in the literature for transverse isotropic materials, and one can thus not conclude on the correctness of the resulting pairing vector. Nonetheless, the results showing good consistency between the reference values and the inverted properties a posteriori justify using the pairing vector to reach our objective.

Our proposal has several limitations that will be addressed in the future. The departure from a 2-D transverse isotropic free plate model that assumes a perfect alignment of the probe with the main bone axis and the assumption of a non absorbing material (when bone is a highly damping one [52]) may affect the accuracy of the retrieved estimates. In addition, the human radius is neither a plate with constant thickness nor a tube with circular cross-section, but rather an irregular hollow shell filled with marrow and surrounded by soft tissue. These factors could conceivably be significant sources of model sophistication (e.g., to account for the overlying soft tissue [53] or a gradient of material properties [54]). In particular, it has been shown that the use of more complex models (e.g., uncoupled bilayer [28] or solid-solid bilayer [29]) can explain additional data arising from the presence of soft tissue. These studies, however, only solved the forward problem using known bone properties, without providing any hints on a possible inversion, i.e., on the ability to infer properties of cortical bone for clinical purposes. It is not clear yet that such multiparametric models could be used in vivo, where both cortical bone and soft tissue properties are unknown, and experimental data are noisy and incomplete. Indeed, a too complex model could lead to overfitting of the data. According to the postulate of the Ockham's razor [55], the simplest model (e.g., a plate model) that is consistent with the data should be favored through a healthy balance between the information gained from the data (i.e., accuracy of the estimates) and the average goodness of fit (i.e., matching between the model and the data) [56]. Consequently, this study does not come down for or against the validity of the plate model in its forward use, but on the correctness of the model parameters inferred by the inverse problem. For the frequency-thickness product range considered here, the obtained estimates close to the reference values suggest that when studied axially and over a relatively short receivers distance, the Lamb wave theory in plates represents a good approximation to explain GWs propagating in the radius, as shown in our previous work $[20,40]$.

Another weakness is related to the empirical nature of the thresholds used in the inversion procedure, that are the 10\%-threshold for the occupancy rate and the euclidean distance $d_{0}$ on the inliers distribution. Both are intimately connected to the maximum allowed uncertainty on the model parameters, which is in this case rather small. Indeed, our approach infers the optimal Lamb modes combination to cover a maximum number of experimental data with an optimum number of Lamb modes, thus defining experimental trajectories in a natural way. In other words, it consists in recognizing a pattern of Lamb modes in the experimental data. Nonetheless, it is rather 
unusual to cope with a given uncertainty on the model parameters, and one commonly wants to quantify it. Consequently, this approach could be refined using a probabilistic approach such as a Bayesian framework [57].

As a further limitation, our approach was tested on a small number of samples compound of ex vivo human specimens and in vivo subjects, whose cortical thickness and porosity cover a range $\left(h \in\left[\begin{array}{ll}2.2 & 3.8\end{array}\right] \mathrm{mm}, p \in\left[\begin{array}{ll}4.1 & 9.2\end{array}\right] \%\right)$, which may likely differ from patients at risk of fractures, in whom thinner cortex and/or higher porosity are expected [5]. In addition, it is known that cortical loss, associated with aging and disease, occurs predominantly in the inner cortex adjacent to the medullary canal [58]. The phenomenon can eventually induce an endosteal resorption, sometimes referred to as cortical bone trabecularization [59], and thinning of the cortex, which will, in turn, lead to irregular inner cortical boundaries (see Figure 2a). Such effect could challenge the hypothesis that cortical bone behaves as a waveguide for ultrasound, particularly in elderly or ill patients. Further studies should be extended to investigate a larger number of subjects, in whom bone properties cover a broader range that might be associated with aging and pathologies. An ex vivo study and a clinical trial on postmenopausal women are currently underway in our group to reach this goal.

Despite these limitations, correctness of thickness and material properties was found to be within few percent on laboratory-controlled samples. Correctness of thickness and porosity was also achieved on ex vivo human specimens. The absolute differences on porosity exceeded those of the thickness, and are expected to be due in part to the assumption of a universal matrix stiffness in the homogenization model. Further modeling efforts are needed to challenge this issue. Although the number of specimens is limited, it is worth pointing out that this study provided the first ex vivo estimates of cortical porosity using the AT technique. In vivo estimates of the cortical thickness were in good agreement with reference values, whereas the porosity estimates could only be compared to literature values. Indeed, there are currently no available technologies to measure cortical porosity or anisotropic elasticity in vivo. As for other US-based technologies (i.e., calcaneal backscatter, shearwave elastography, etc.), a surrogate for tissue elasticty cannot be directly validated in vivo but requires previous calibration on phantoms and ex vivo validation studies. Then, its performance is tested in vivo on a large number of subjects based on criteria of clinical interest. As our approach was validated on phantoms and ex vivo, we believe that the in vivo prospective results presented here are of interest for the community and represent the first feasibility step for future clinical trials, although its clinical value is beyond the scope of the present work. A strength of our proposal is that it might be straightforwardly extended to a multidimensional optimization (i.e., four elastic coefficients instead of the porosity), and a face-to-face comparison between GWs-based characterization of bone specimens and resonant ultrasound spectroscopy estimation constitutes one of the next step of this study. 


\section{Conclusion}

This study reported on the use of an efficient optimization method for estimating both the cortical thickness and porosity of cortical bone. The proposed method automatically takes into account the optimal combination of Lamb modes that are necessary to explain the data by including an additional model parameter in the inverse procedure, thus avoiding pairing burden between the incomplete data and the Lamb modes. Genetic algorithms were used as search algorithms due to their capability of finding a near global solution where the objective function is multidimensional and non-convex. This method was first validated on laboratory-controlled measurements performed on isotropic plates and bone-mimicking phantoms. Then, it was successfully applied to a set of ex vivo and in vivo data, providing estimates of cortical thickness and porosity that were in good agreement with reference values derived from X-ray computed tomography.

\section{Acknowledgment}

This research was supported by the Fondation pour la Recherche Médicale through project number FRM DBS201311228444 and AZALEE. Authors would like to thank Dr. Maryline Talmant for her technical support and the fruitful discussions throughout this study. Authors also would like to thank Dr. David Mitton and Rémy Gauthier for providing us the bone samples, and Dr. Christine Chappard for the HR-pQCT images.

\section{References}

[1] E Jansons, A Tatarinov, V Dzenis, and A Kregers. Constructional peculiarities of the human tibia defined by reference to ultrasound measurement data. Biomaterials, 5(4):221-226, 1984.

[2] PHF Nicholson, P Moilanen, T Kärkkäinen, J Timonen, and S Cheng. Guided ultrasonic waves in long bones: modelling, experiment and in vivo application. Physiological measurement, 23(4):755-768, 2002.

[3] F Lefebvre, Y Deblock, P Campistron, D Ahite, and JJ Fabre. Development of a new ultrasonic technique for bone and biomaterials in vitro characterization. Journal of biomedical materials research, 63(4):441-446, 2002.

[4] M Talmant, J Foiret, and J-G Minonzio. Guided waves in cortical bone. In Bone Quantitative Ultrasound, pages 147-179. Springer, 2011.

[5] KK Nishiyama, HM Macdonald, HR Buie, DA Hanley, and SK Boyd. Postmenopausal women with osteopenia have higher cortical porosity and thinner cortices at the distal radius and tibia than women with normal abmd: an in vivo hr-pqct study. Journal of Bone and Mineral Research, 25(4):882-890, 2010.

[6] P Moilanen, PHF Nicholson, V Kilappa, S Cheng, and J Timonen. Assessment of the cortical bone thickness using ultrasonic guided waves: Modelling and in vitro study. Ultrasound in medicine E biology, 33(2):254-262, 2007.

[7] E Camus, M Talmant, G Berger, and P Laugier. Analysis of the axial transmission technique for the assessment of skeletal status. The Journal of the Acoustical Society of America, 108(6):3058$3065,2000$.

[8] E Bossy, M Talmant, F Peyrin, L Akrout, P Cloetens, and P Laugier. An in vitro study of the ultrasonic axial transmission technique at the radius: $1-\mathrm{mhz}$ velocity measurements are sensitive 
to both mineralization and intracortical porosity. Journal of Bone and Mineral Research, 19(9):1548-1556, 2004.

[9] MR Stegman, RP Heaney, D Travers-Gustafson, and J Leist. Cortical ultrasound velocity as an indicator of bone status. Osteoporosis international, 5(5):349-353, 1995.

[10] D Hans, SK Srivastav, C Singal, R Barkmann, CF Njeh, E Kantorovich, CC Glüer, and HK Genant. Does combining the results from multiple bone sites measured by a new quantitative ultrasound device improve discrimination of hip fracture? Journal of Bone and Mineral Research, 14(4):644-651, 1999.

[11] R Barkmann, E Kantorovich, C Singal, D Hans, HK Genant, M Heller, and C-C Glüer. A new method for quantitative ultrasound measurements at multiple skeletal sites: first results of precision and fracture discrimination. Journal of Clinical Densitometry, 3(1):1-7, 2000.

[12] M Weiss, AB Ben-Shlomo, P Hagag, and M Rapoport. Reference database for bone speed of sound measurement by a novel quantitative multi-site ultrasound device. Osteoporosis International, 11(8):688-696, 2000.

[13] M Talmant, S Kolta, C Roux, D Haguenauer, I Vedel, B Cassou, E Bossy, and P Laugier. In vivo performance evaluation of bi-directional ultrasonic axial transmission for cortical bone assessment. Ultrasound in medicine $\mathcal{E}$ biology, 35(6):912-919, 2009.

[14] P Moilanen, M Määttä, V Kilappa, L Xu, PHF Nicholson, M Alén, J Timonen, T Jämsä, and S Cheng. Discrimination of fractures by low-frequency axial transmission ultrasound in postmenopausal females. Osteoporosis International, 24(2):723-730, 2013.

[15] V Egorov, A Tatarinov, N Sarvazyan, R Wood, L Magidenko, S Amin, S Khosla, RJ Ruh, JM Ruh, and A Sarvazyan. Osteoporosis detection in postmenopausal women using axial transmission multi-frequency bone ultrasonometer: clinical findings. Ultrasonics, 54(5):1170-1177, 2014.

[16] P Moilanen, PHF Nicholson, V Kilappa, S Cheng, and J Timonen. Measuring guided waves in long bones: Modeling and experiments in free and immersed plates. Ultrasound in medicine $\mathcal{G}^{3}$ biology, 32(5):709-719, 2006.

[17] A Tatarinov, N Sarvazyan, and A Sarvazyan. Use of multiple acoustic wave modes for assessment of long bones: Model study. Ultrasonics, 43(8):672-680, 2005.

[18] P Moilanen. Ultrasonic guided waves in bone. IEEE Transactions on Ultrasonics, Ferroelectrics, and Frequency Control, 55(6):1277-1286, 2008.

[19] D Ta, W Wang, YY Wang, LH Le, and Y Zhou. Measurement of the dispersion and attenuation of cylindrical ultrasonic guided waves in long bone. Ultrasound in medicine ${ }^{3}$ biology, 35(4):641$652,2009$.

[20] J Foiret, J-G Minonzio, C Chappard, M Talmant, and P Laugier. Combined estimation of thickness and velocities using ultrasound guided waves: A pioneering study on in vitro cortical bone samples. IEEE Transactions on Ultrasonics, Ferroelectrics, and Frequency Control, 61(9):1478$1488,2014$.

[21] WP Rogers. Elastic property measurement using rayleigh-lamb waves. Research in Nondestructive Evaluation, 6(4):185-208, 1995.

[22] MR Karim, AK Mal, and Y Bar-Cohen. Inversion of leaky lamb wave data by simplex algorithm. The Journal of the Acoustical Society of America, 88(1):482-491, 1990.

[23] JL Deán, C Trillo, ÁF Doval, and JL Fernández. Determination of thickness and elastic constants of aluminum plates from full-field wavelength measurements of single-mode narrowband lamb waves. The Journal of the Acoustical Society of America, 124(3):1477-1489, 2008.

[24] L Yan, H Cunfu, S Guorong, W Bin, C-H Chung, and Y-C Lee. Elastic properties inversion of an isotropic plate by hybrid particle swarm-based-simulated annealing optimization technique from leaky lamb wave measurements using acoustic microscopy. Journal of Nondestructive Evaluation, 33(4):651-662, 2014.

[25] IA Veres and MB Sayir. Wave propagation in a wooden bar. Ultrasonics, 42(1):495-499, 2004.

[26] D Gsell and J Dual. Non-destructive evaluation of elastic material properties in anisotropic circular cylindrical structures. Ultrasonics, 43(2):123-132, 2004. 
[27] A Marzani and L De Marchi. Characterization of the elastic moduli in composite plates via dispersive guided waves data and genetic algorithms. Journal of Intelligent Material Systems and Structures, 24(17):2135-2147, 2013.

[28] J Chen, J Foiret, J-G Minonzio, M Talmant, Z Su, L Cheng, and P Laugier. Measurement of guided mode wavenumbers in soft tissue-bone mimicking phantoms using ultrasonic axial transmission. Physics in medicine and biology, 57(10):3025-3037, 2012.

[29] TNHT Tran, L Stieglitz, YJ Gu, and LH Le. Analysis of ultrasonic waves propagating in a bone plate over a water half-space with and without overlying soft tissue. Ultrasound in medicine 83 biology, 39(12):2422-2430, 2013.

[30] M Maraschini, F Ernst, S Foti, and LV Socco. A new misfit function for multimodal inversion of surface waves. Geophysics, 75(4):G31-G43, 2010.

[31] J Vishnuvardhan, CV Krishnamurthy, and K Balasubramaniam. Genetic algorithm based reconstruction of the elastic moduli of orthotropic plates using an ultrasonic guided wave single-transmitter-multiple-receiver shm array. Smart Materials and Structures, 16(5):1639$1650,2007$.

[32] J Vishnuvardhan, CV Krishnamurthy, and K Balasubramaniam. Blind inversion method using lamb waves for the complete elastic property characterization of anisotropic plates. The Journal of the Acoustical Society of America, 125(2):761-771, 2009.

[33] X Song and $\mathrm{H} \mathrm{Gu}$. Utilization of multimode surface wave dispersion for characterizing roadbed structure. Journal of Applied Geophysics, 63(2):59-67, 2007.

[34] Tables of Physical \& Chemical Constants. 2.4.1 The speed and attenuation of sound. Kaye \& Laby Online, 2005.

[35] S Bernard, Q Grimal, S Haupert, and P Laugier. Assessment of anisotropic elasticity of small bone samples with resonant ultrasound spectroscopy: Attenuation does not prevent the measurements. In IEEE International Ultrasonics Symposium, pages 1599-1602. IEEE, 2011.

[36] D Ulrich, B Van Rietbergen, A Laib, and P Ruegsegger. The ability of three-dimensional structural indices to reflect mechanical aspects of trabecular bone. Bone, 25(1):55-60, 1999.

[37] L Moreau, J-G Minonzio, J Foiret, E Bossy, M Talmant, and P Laugier. Accurate measurement of guided modes in a plate using a bidirectional approach. The Journal of the Acoustical Society of America, 135(1):EL15-EL21, 2014.

[38] J-G Minonzio, M Talmant, and P Laugier. Guided wave phase velocity measurement using multiemitter and multi-receiver arrays in the axial transmission configuration. The Journal of the Acoustical Society of America, 127(5):2913-2919, 2010.

[39] J-G Minonzio, J Foiret, M Talmant, and P Laugier. Impact of attenuation on guided mode wavenumber measurement in axial transmission on bone mimicking plates. The Journal of the Acoustical Society of America, 130(6):3574-3582, 2011.

[40] J-G Minonzio, J Foiret, P Moilanen, J Pirhonen, Z Zhao, M Talmant, J Timonen, and P Laugier. A free plate model can predict guided modes propagating in tubular bone-mimicking phantoms. The Journal of the Acoustical Society of America, 137(1):EL98-EL104, 2015.

[41] S-H Rhee, J-K Lee, and J-J Lee. The group velocity variation of lamb wave in fiber reinforced composite plate. Ultrasonics, 47(1):55-63, 2007.

[42] M Granke, Q Grimal, A Saïed, P Nauleau, F Peyrin, and P Laugier. Change in porosity is the major determinant of the variation of cortical bone elasticity at the millimeter scale in aged women. Bone, 49(5):1020-1026, 2011.

[43] Q Grimal and WJ Parnell. Cortical bone homogenization. Retrieved April 5, 2016 from https://www.lib.upmc.fr/ qgrimal/downloads.html.

[44] W J Parnell and Q Grimal. The influence of mesoscale porosity on cortical bone anisotropy. investigations via asymptotic homogenization. Journal of the Royal Society Interface, 6(30):97109, 2009.

[45] WJ Parnell, MB Vu, Q Grimal, and S Naili. Analytical methods to determine the effective mesoscopic and macroscopic elastic properties of cortical bone. Biomechanics and modeling in 
mechanobiology, 11(6):883-901, 2012.

[46] D Goldberg. Genetic algorithms in search, optimization and machine learning. Addison-Wesley Publ. (Reading, Massachussets), 1989.

[47] K Sun, K Hong, L Yuan, Z Shen, and X Ni. Inversion of functional graded materials elastic properties from ultrasonic lamb wave phase velocity data using genetic algorithm. Journal of Nondestructive Evaluation, 33(1):34-42, 2014.

[48] AA Fahim, R Gallego, N Bochud, and G Rus. Model-based damage reconstruction in composites from ultrasound transmission. Composites Part B: Engineering, 45(1):50-62, 2013.

[49] S Bernard, G Marrelec, P Laugier, and Q Grimal. Bayesian normal modes identification and estimation of elastic coefficients in resonant ultrasound spectroscopy. Inverse Problems, 31(6):065010, 2015.

[50] DML Cooper, JR Matyas, MA Katzenberg, and B Hallgrimsson. Comparison of microcomputed tomographic and microradiographic measurements of cortical bone porosity. Calcified tissue international, 74(5):437-447, 2004.

[51] W Tjong, J Nirody, A J Burghardt, J Carballido-Gamio, and GJ Kazakia. Structural analysis of cortical porosity applied to hr-pqct data. Medical physics, 41(1):013701, 2014.

[52] G Haïat. Linear ultrasonic properties of cortical bone: In vitro studies. In Bone Quantitative Ultrasound, pages 331-360. Springer, 2011.

[53] P Moilanen, M Talmant, V Kilappa, P Nicholson, S Cheng, J Timonen, and P Laugier. Modeling the impact of soft tissue on axial transmission measurements of ultrasonic guided waves in human radius. The Journal of the Acoustical Society of America, 124(4):2364-2373, 2008.

[54] G Haïat, S Naili, Q Grimal, M Talmant, C Desceliers, and C Soize. Influence of a gradient of material properties on ultrasonic wave propagation in cortical bone: application to axial transmission. The Journal of the Acoustical Society of America, 125(6):4043-4052, 2009.

[55] SF Gull. Developments in maximum entropy data analysis. In Maximum entropy and Bayesian methods, pages 53-71. Springer, 1989.

[56] N Bochud and G Rus. Probabilistic inverse problem to characterize tissue-equivalent material mechanical properties. IEEE Transactions on Ultrasonics, Ferroelectrics, and Frequency Control, 59(7):1443-1456, 2012.

[57] A Tarantola. Inverse problem theory and methods for model parameter estimation. Society for Industrial and Applied Mathematics, 2005.

[58] Y Bala, R Zebaze, and E Seeman. Role of cortical bone in bone fragility. Current opinion in rheumatology, 27(4):406-413, 2015.

[59] AM Parfitt. Age-related structural changes in trabecular and cortical bone: cellular mechanisms and biomechanical consequences. Calcified Tissue International, 36(1):S123-S128, 1984. 